\title{
NATIONWIDE NATURAL RESOURCE INVENTORY OF THE PHILIPPINES USING LIDAR: STRATEGIES, PROGRESS, AND CHALLENGES
}

\author{
A. C. Blanco ${ }^{\text {a* }}$, A. Tamondong ${ }^{\text {a }}$, A.M. Perez ${ }^{\text {a }}$, M.R.C. Ang $^{\text {a }}$, E. Paringit ${ }^{\text {a }}$, R. Alberto ${ }^{\text {b }}$, N. Alibuyog ${ }^{\text {c }}$, D. Aquino ${ }^{\text {d }}$, A. Ballado ${ }^{\text {e }}$, P.

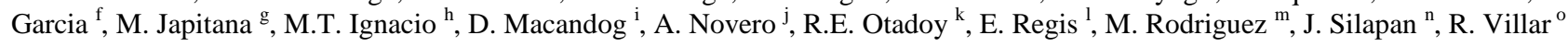 \\ ${ }^{a}$ Dept. of Geodetic Engineering, Training Center for Applied Geodesy and Photogrammetry, University of the Philippines, Diliman, \\ Quezon City, Philippines - *ariel.blanco@coe.upd.edu.ph \\ ${ }^{\mathrm{b}}$ Central Luzon State University, ${ }^{\mathrm{c}}$ Mariano Marcos State University, ${ }^{\mathrm{d}}$ Isabela State University, ${ }^{\mathrm{e}}$ Mapua Institute of Technology, ${ }^{\mathrm{f}}$ \\ Visayas State University, ${ }^{\mathrm{g}}$ Caraga State University, ${ }^{\mathrm{h}}$ Mindanao State University - Iligan Institute of Technology, \\ ${ }^{\mathrm{i}}$ University of the Philippines Los Banos, ${ }^{\mathrm{j}}$ University of the Philippines Mindanao, ${ }^{\mathrm{k}}$ University of San Carlos, \\ ${ }^{1}$ Ateneo de Naga University, ${ }^{\mathrm{m}}$ Ateneo de Zamboanga University, ${ }^{\mathrm{n}}$ University of the Philippines Cebu, ${ }^{\circ}$ Central Mindanao \\ University
}

\section{Commission VI, WG VI/6}

KEY WORDS: LiDAR, natural resources, agricultural, forest, coastal, hydrological, renewable energy

\begin{abstract}
:
The Philippines has embarked on a detailed nationwide natural resource inventory using LiDAR through the Phil-LiDAR 2 Program. This 3-year program has developed and has been implementing mapping methodologies and protocols to produce high-resolution maps of agricultural, forest, coastal marine, hydrological features, and renewable energy resources. The Program has adopted strategies on system and process development, capacity building and enhancement, and expanding the network of collaborations. These strategies include training programs (on point cloud and image processing, GIS, and field surveys), workshops, forums, and colloquiums (program-wide, cluster-based, and project-based), and collaboration with partner national government agencies and other organizations. In place is a cycle of training, implementation, and feedback in order to continually improve the system and processes. To date, the Program has achieved progress in the development of workflows and in rolling out products such as resource maps and GIS data layers, which are indispensable in planning and decision-making. Challenges remains in speeding up output production (including quality checks) and in ensuring sustainability considering the short duration of the program. Enhancements in the workflows and protocols have been incorporated to address data quality and data availability issues. More trainings have been conducted for project staff hired to address human resource gaps. Collaborative arrangements with more partners are being established. To attain sustainability, the Program is developing and instituting a system of training, data updating and sharing, information utilization, and feedback. This requires collaboration and cooperation of the government agencies, LGUs, universities, other organizations, and the communities.
\end{abstract}

\section{NATURAL RESOURCE MAPPING IN THE PHILIPPINES}

The mapping and inventory of natural resources in the Philippines are undertaken by various national government agencies (NGAs) and other organizations such as nongovernmental organizations (NGOs) or non-profit organizations (NPOs). Nationwide mapping is typically carried out by NGAs periodically. Perhaps the most frequent is every five (5) years. Most of the resource maps are based on moderate-resolution satellite images and therefore, the classification level may not be enough for detailed planning needs of local government units. Methodologies used and, therefore, resulting accuracies of maps vary greatly. In addition, documentation of methodologies and details on classification accuracy and map quality assessments are mostly not available. The availability of these resource maps is also limited. Thus, there is the need to come up with resource maps with sufficient detail to support various planning and development needs. In this paper, the utilization of LiDAR datasets for nationwide natural resource mapping is presented.

\footnotetext{
* Corresponding author
}

\section{OVERVIEW OF THE PHIL-LIDAR 2 PROGRAM}

\subsection{Program Objectives}

The Phil-LiDAR 2 Program started as an offshoot program of the DREAM (Disaster Risk Exposure and Assessment for Mitigation) Program of the University of the Philippines and the Department of Science and Technology (DOST). With the availability of LiDAR datasets, the Phil-LiDAR 2 Program aims to provide detailed resource inventory using LiDAR and other remotely sensed data. The program is tasked to map the following resources for the entire Philippines: agricultural, forest, coastal, water, and renewable energy.

The Program complements on-going programs of government agencies by utilizing LIDAR data to produce high-resolution natural resource maps and vulnerability assessments of agricultural and coastal marine resources. This requires the development of methodologies for extracting resource features from LiDAR and other RS data. The Program is also tasked to 
formulate recommendations to help address future local supply and demand in agriculture, coastal, forest, and renewable energy resources.

\subsection{Program Implementation}

The 3-year Program is being implemented since July 2014 with the University of the Philippines Diliman (UPD), through its Training Center for Applied Geodesy and Photogrammetry, leading fourteen (14) other State Universities and Colleges (SUCs) and Higher Educational Institutes (HEIs) throughout the Philippines. The SUCs and HEIs are clustered into three, representing the major groups of islands in the Philippines: Luzon cluster, Visayas cluster, and Mindanao cluster. The core methodological workflows are developed by UPD and these are cascaded to the SUCs and HEIs for implementation and enhancement as needed to address peculiarities in land cover in their assigned areas. SUCs and HEIs undertake field validation and accuracy assessment activities and then submit the outputs to UPD for the second-level of quality assurance. Products are distributed to user once standards of accuracy and map quality are met.

There are five (5) core projects at UPD as listed:

a. Project 1. Agricultural Resources Assessment using LIDAR (PARMap)

b. Project 2. Coastal Resources Assessment using LIDAR (CoastMap)

c. Project 3. Forest Resources Assessment using LIDAR (FRExLS)

d. Project 4. Development of the Philippine Hydrologic Datasets (PHD) for Watersheds using LIDAR

e. Project 5. Renewable Energy Resources Mapping (REMaps) using LIDAR

Each SUC/HEI implements all or some of the resource mapping tasks in close coordination with the above-listed projects.

\section{RESOURCE MAPPING AND INVENTORY STRATEGIES}

Undertaking a nationwide natural resource mapping and inventory utilizing LiDAR in relatively short period of time and considering various constraints and challenges requires the formulation and adoption of strategies to ensure program objectives are met. From the start, the following are recognized:

a. There are only a few universities with experts in mapping and remote sensing. In addition, there exist only a few courses which provide academic training in Geomatics.

b. There are other on-going efforts of various agencies and groups to map at various scales the natural resources using various datasets and methodology.

Considering the above, following key strategies were laid out and implemented:

a. Training, mentoring, and monitoring responsive to program needs;

b. Delineation of responsibilities considering strengths and weaknesses;

c. Knowledge sharing and consensus building through various activities; d. Establishment and strengthening of collaboration and cooperation; and

e. Building and ensuring sustainability through various means.

\subsection{Training, mentoring, and monitoring}

Training programs have been developed to address the lack of formal training on the use of geospatial technologies for mapping natural resources. While the methodological workflows were being developed, training on basic remote sensing and GIS was provided to project staff. Workflow- and task-specific trainings were also provided after the development of workflows and protocols (e.g., field validation). Mentoring activities ensure that knowledge and skills acquired through the trainings are enhanced and applied properly. Monitoring is both conducted by the UPD teams (focusing on technical aspects) and by the DOST-PCIEERD team (focusing on administrative aspects) to ensure successful implementation of the project tasks.

\subsection{Delineation of responsibilities}

Crucial also is the clear identification of project areas considering the physical location of the universities, natural resource types in the regions, and specialization of the university teams. This means that project area assignments need not follow the regional administrative groupings but rather logistical efficiency and prior work in the areas must be taken into consideration. In addition to the assignment of areas, the selection and assignment of resource types to be mapped by each university, including UPD, were conducted through discussions. The delineation of responsibilities included also the collaboration between universities. For examples, UPD processes the LiDAR data and universities do the field validation.

\subsection{Knowledge sharing and consensus building}

The composition of project teams under the Program varies considerably due to availability of varied expertise or specialization in the universities and the regions. This presents an opportunity for synergy of ideas from different but related fields and disciplines. Phil-LiDAR 2 undertakes program-wide and cluster-level colloquia to report progress, experiences, and issues in project implementation and discuss solutions. These are opportunities to resolve problems at various levels and forge consensus on various aspects of the Program. The Program also conducts symposia, fora, and workshops as venues for other experts and partners to share their knowledge and experiences.

\subsection{Collaboration and cooperation}

Resource mapping is considered collaborative undertaking wherein support and assistance from various stakeholders are necessary to ensure the quality and usability of maps and GIS layers. Collaboration and cooperation includes field validation, provision of secondary data, logistical support, feedbacks on project outputs, and other activities. Collaboration and cooperation are also means to share knowledge and skills developed in the implementation of the projects.

In order to strengthen collaboration with LGUs, NGAs, and other partners, the Program adopts a quick deployment of maps after quality standards are met. This strategy has enabled the 
Program to gain the trust of organizations, which translated into greater support to the SUCs/HEIs implementing the projects.

\subsection{Building and ensuring sustainability}

In this last year of the Program, activities are geared towards building and ensuring sustainability of project activities. Collaboration and cooperation serve as cornerstone of sustainability as some of project activities have to be devolved eventually to agencies, LGUs, and partners. It is envisioned that the universities would provide technical assistance to LGUs and other organizations. Some details on activities to attain sustainability are presented in section 5 .

\section{PROGRESS AND GAINS UNDER THE PROGRAM}

The DOST-PCIEERD evaluates R\&D projects using the 5Ps, namely, Products, People Service, Partnerships, Publications, and Patent/Potential IP. The progress and gains under the PhilLiDAR 2 Program is presented in this section following these criteria.

\subsection{Products: Workflows and Maps}

The Phil-LiDAR 2 Program was able to develop core workflows in as early as six months of project implementation. These workflows cover the following processes:

a. Agricultural land cover and land use mapping using LiDAR and other RS data,

b. Benthic cover (corals, seagrass, macroalgae), mangrove, aquaculture and mariculture mapping,

c. Forest cover, canopy height, biomass, carbon stock assessment,

d. Extraction of various hydrological features and establishment of the Philippine Hydrologic Dataset,

e. Assessment of biomass, hydropower, solar energy, and wind energy at national and urban scales.

The various workflows primarily utilize OBIA and SVM for classification. Workflows for map generalization and composition have been developed and deployed as well. The workflows make use of the following software: LAStools, eCognition, ENVI, ERDAS Imagine, Mathlab, ArcGIS, QGIS, and GeoMedia. Figures 1 to 4 show sample maps for hydropower resource assessment, agricultural land cover, combined agricultural and coastal land cover, and streams and catchments, respectively.

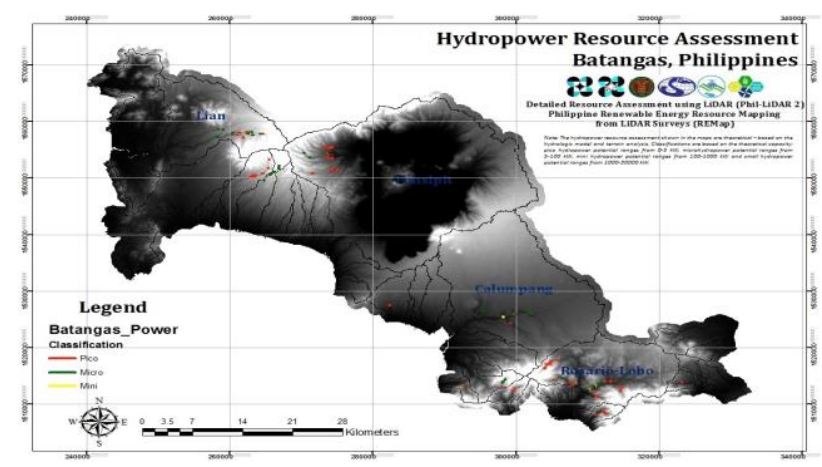

Figure 1. Sample hydropower assessment map

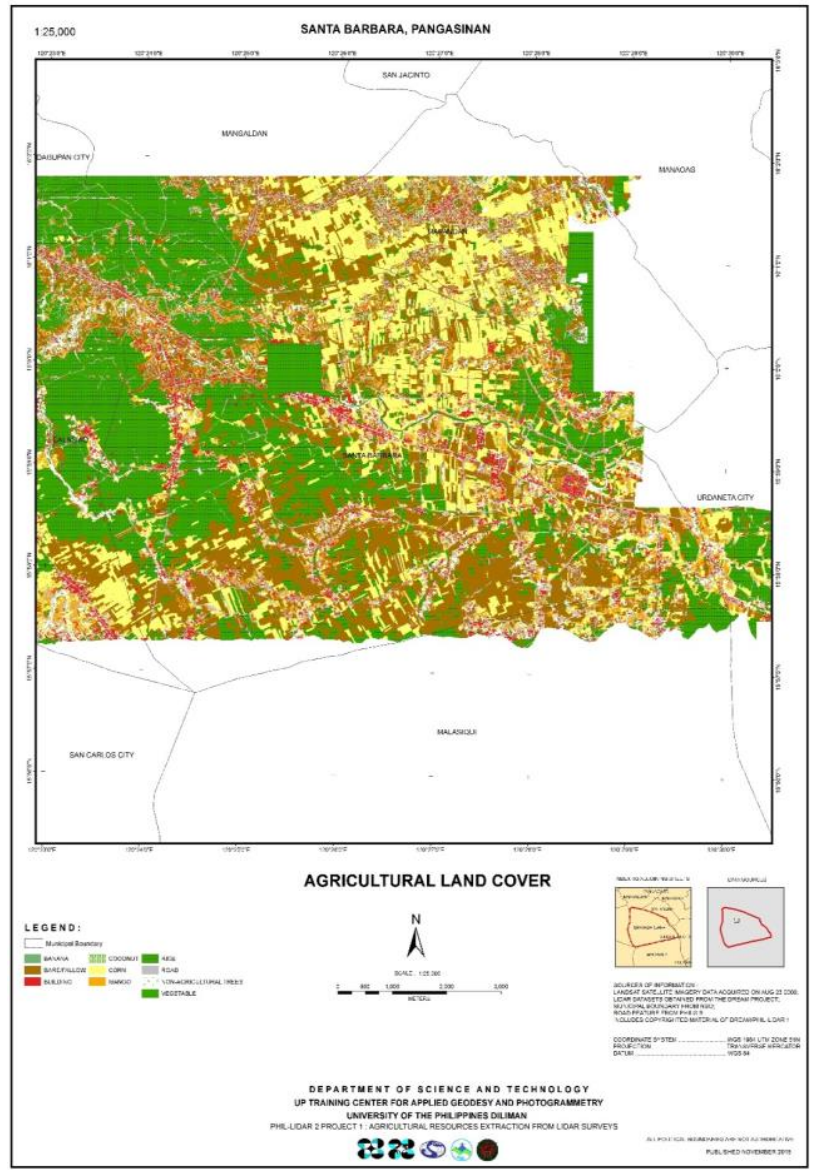

Figure 2. Sample 1:10,000 agricultural land cover map

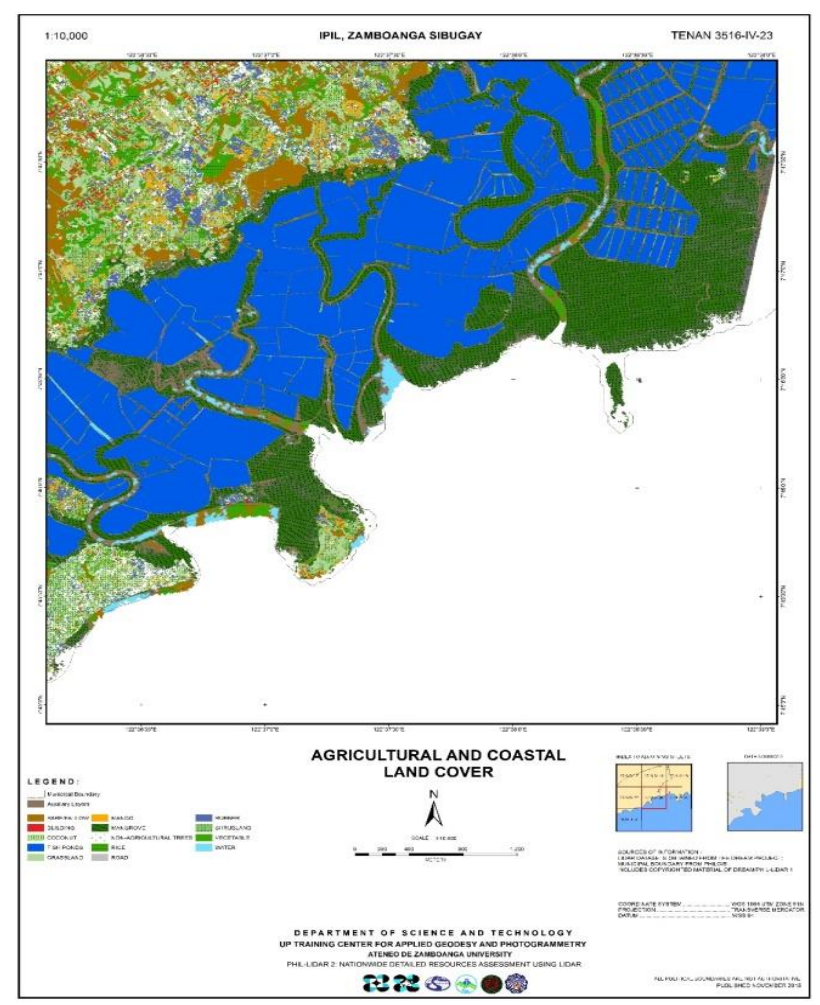

Figure 3. Sample agricultural and coastal land cover map 


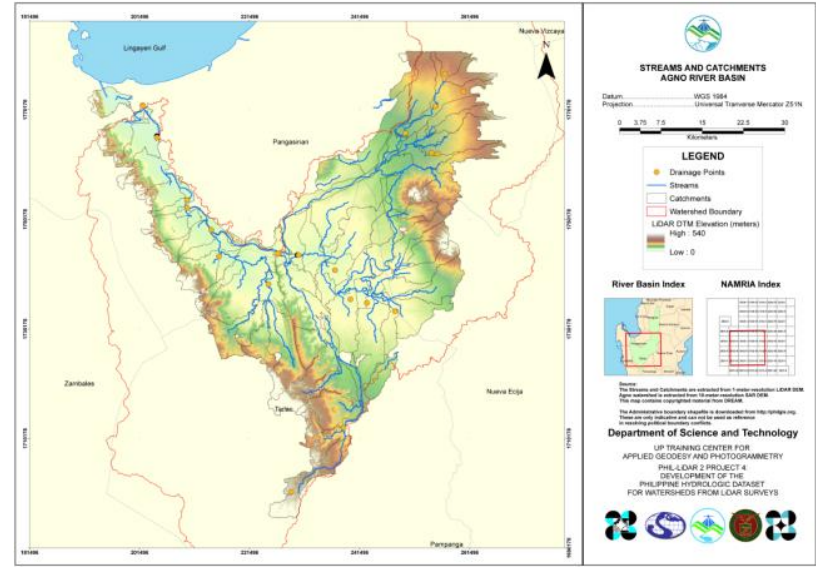

Figure 4. Sample streams and catchments map

As of December 2015, the Program has distributed the following through map turn-over ceremonies conducted at the program-level (Figure 5):
a. 107 municipal/city agricultural land cover maps
b. 34 municipal coastal resource maps
c. 2 coastal resource maps for national government agencies
d. 6 forest resource maps covering plot sites
e. 41 PHD maps covering 16 river basins
f. 3 provincial hydropower assessment maps

To further facilitate the distribution of maps and GIS layers to more stakeholders, turn-over activities are now conducted by each university. In all these activities, the universities are required to conducted orientation, forum, and short course training for LGUs and other stakeholders. This aims to ensure that users are aware of the intended usage as well as the limitations of the maps. The LGUs in particular are encouraged and trained to update the GIS layers periodically.

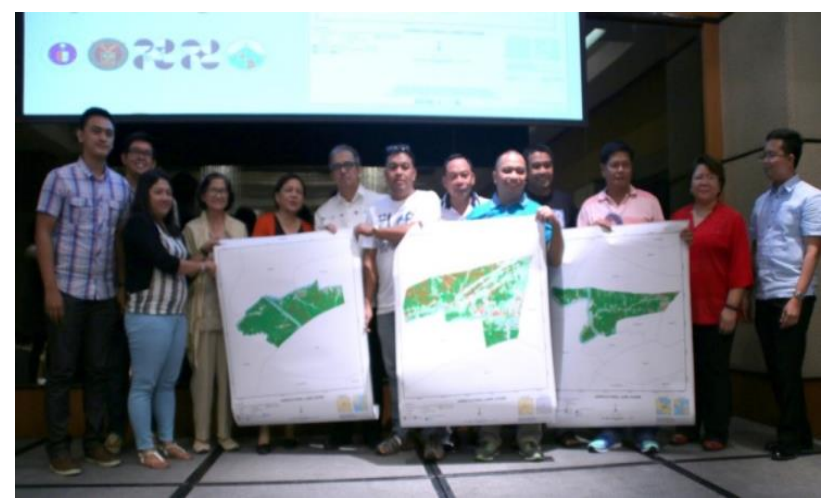

Figure 5. Turn-over of Phil-LiDAR 2 Agricultural Cover Maps to local government units

\subsection{People Service}

The Phil-LiDAR 2 Program capacitated researchers and engineers to utilize LiDAR and other remote sensing/GIS data for resource inventory. A total of 769 researchers and experts were trained under the Program. It is considered first of its kind and the biggest collaboration of multidisciplinary researchers and experts from public and private universities in the Philippines. With the capability shared throughout the country, it is envisioned that human resources would be sustained by developing regional hubs of LiDAR data processing and mapping services. These will address the needs of the local communities for accurate and updated geospatial information. Local government officials can formulate science-based policies to address and sustain local supply and demand in agriculture, coastal marine, forest, water, and renewable energy resources. The provision of detailed resource maps is expected to improve monitoring, planning, and management of the country's natural resources.

\subsection{Partnerships}

Collaborative partnership has been established with relevant national government agencies, including their bureaus and regional offices. These include the Department of Agriculture, Department of Energy, Department of Environment and Natural Resources, National Irrigation Administration, and the National Water Resource Board.

Various collaborative arrangements are in place. In addition to the NGAs, partners of the Program include LGUs, other SUCs/HEIs, and other organizations such as GIZ, WWF and the Philippine Eagle Foundation.

\subsection{Conference Presentations and Publications}

The R\&D outputs of the Program have been presented in various local and international conferences. More than 15 local conference papers and more than 50 international conference papers were presented. Two papers have been accepted for publication in the ISPRS Annals and many others have been accepted for publication in the ISPRS Archives.

\subsection{Patent/Potential IP}

Two android applications have been developed by the Ateneo de Naga University (AdNU) and Caraga State University. Figure 6 shows the screen capture of AdNU's reGIS app, which features map, data dictionary, coordinate averaging, autoupdating of collected data by field personnel in the survey area.

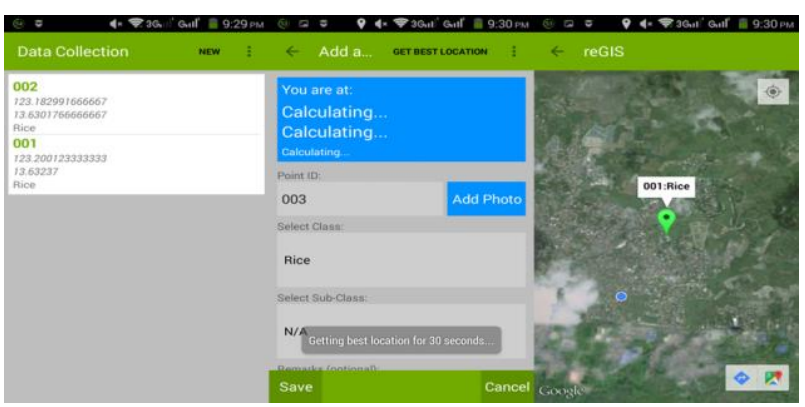

Figure 6. AdNU's reGIS app for field data collection

\section{CHALLENGES IN RESOURCE ASSESSMENT}

Natural resource assessment is viewed by the Phil-LiDAR 2 Program as integrated effort by all stakeholders, including the project staff, LGUs, agencies, communities, and other. As such, challenges identified include those within the implementation of the program and those after the completion of the program. This section presents these challenges and provides insight and actions to address them. 


\subsection{Implementation Challenges}

Within program implementation, the generation of outputs have to be sped up considering the limited number of project years. In the second year of implementation, new research assistants were employed. This meant giving them trainings and providing them ample time to digest the new knowledge and hone their skills. This time varies depending on the academic background and work experience of the new research assistants. Considering that only a few courses have mapping subjects, some difficulties have been encountered. Some who have been trained before are not yet capable to be trainers given quick pace of knowledge acquisition and learning by application or practice. This has been overcome by (1) conducting program-level training sessions, (2) streamlining process and enhancing task assignments, and (3) undertaking detailed performance evaluation.

There is also the task of continuously enhancing the workflows to address difficulties in obtaining acceptable accuracies in some of the project sites. This is brought about by the variability in data availability (e.g., no orthomosaic) and some issues in data quality (e.g., LiDAR intensity). This has been addressed by employing additional derivative layers from the LiDAR point clouds and conducting normalization of LiDAR intensity across flight lines. If the latter did not work, processing is carried out by flight lines.

\subsection{Post-program Challenges and Attaining Sustainability}

Mark footnotes in the text with a number (1); use the same number for a second footnote of the paper and so on. Place footnotes at the bottom of the page, separated from the text above it by a horizontal line.

Strategies and plans for sustainability include the following:

a. New R\&D projects

Initially identified projects include crop suitability mapping, vulnerability and risk assessment and mapping, development of decision support tools for land use planning, soil and water quality assessment using LiDAR induced fluorescence and hyperspectral imaging, pollution mapping, urban expansion mapping, GIS mapping of crop health, infestations, and contaminations

b. Extension services by universities

These cover support in updating of resource maps, training, seminars, and workshops, GIS and data regional hub

c. Collaboration with LGUs and government agencies Possible collaboration includes information dissemination to farmers and other groups, assistance in reforestation projects, and support in the preparation of comprehensive land use plans.

d. Collaboration within university

Some universities are eyeing the creation of university geoinformatics centers. Trainings for faculty, research staff, and students will be conducted. Continuous data collection using meteorological sensors deployed will be done. Joint project such as coastal resource management will be explored. The use of remote sensing and GIS in other research projects and thesis work are being promoted.

e. University positions

Researcher and lecturer positions are available in some SUCs/HEIs.

f. Other activities

Other activities include the organization of symposia, colloquia, forums as well as continuous GIS capability building for communities.

\section{SUMMARY AND CONCLUSIONS}

The Phil-LiDAR 2 Program, consisting of 15 universities, has achieved significant progress not only in the development of workflows and protocols but also in human resource development and capacity building. A mechanism for data gathering, data processing, quality check and coordination at different levels is in place to ensure that program and project objectives are attained. Activities for ensuring sustainability of the various activities have been identified. Continuous collaboration and cooperation among all stakeholders are seen as the way to continue the gains made under the Phil-LiDAR.

\section{ACKNOWLEDGEMENTS}

The authors are thankful for the funds provided by the Philippines' Department of Science and Technology (DOST), for the management support by the DOST Philippine Council for Industry, Energy and Emerging Technology Research and Development (DOST-PCIEERD) and for the dedicated work of all Phil-LiDAR 2 researchers. The authors are grateful for the support of the DREAM Program and Phil-LiDAR 2 Program. Special thanks go to Dr. Martin Isenburg for providing additional training and mentoring of project staff. 